\title{
Türkiye’den Bazı Kareler İçin Yeni Floristik Kayıtlar
}

\author{
Lütfi BEHÇET, Yakup YAPAR* \\ Bingöl Üniversitesi, Fen-Edebiyat Fakültesi, Biyoloji Bölümü, Bingöl \\ (ORCID: 0000-0001-8334-7816) (ORCID: 0000-0002-5298-0085)
}

\begin{abstract}
$\ddot{O} \mathbf{z}$
Bu çalışmada, çeşitli kareler (A5, B7, B8, B9) için yeni 77 bitki taksonunun yayllı̧̧ı ilk defa verilmektedir. Bu taksonlardan 13'ü (Bunium microcarpum (Boiss.) Freyn subsp. longiradiatum Hedge \& Lamond, Trigonosciadium intermedium Freyn \& Sint., Tanacetum eginense (Hausskn. ex Bornm.) Grierson, Heldreichia bupleurifolia Boiss. subsp. rotundifolia (Boiss.) Parolly, Nordt \& Mumm. var. rotundifolia, Hesperis pisidica Hub.-Mor., Anchusa limbata Boiss., Euonymus Iatifolius (L.) Mill. subsp. cauconis Coode \& Cullen, Astragalus nitens Boiss. \& Heldr., Salvia cilicica Boiss. \& Kotschy, S. euphratiaca Montbret \& Aucher ex Bentham var. leiocelycina (Rech.f.) Hedge, Stachys setifera C.A.Mey. subsp. lycia (Gand.) Bhattacherje, Rhynchocorys odontophylla R. B. Burb \& I. Richardson, Acantholimon calvertii Boiss. var. glabrum Akaydın et Doğan) endemiktir. Belirlenen taksonların 8 tanesinde floradaki tanımlarından farklı bazı varyasyonlar tespit edilmiştir. Bu çalışmada verilen 77 taksonun 19'u İran-Turan, 10'u Akdeniz, 8'i Avrupa Sibirya fitocoğrafik bölge elementi iken; 40’1 çok bölgeli veya fitocoğrafik bölgesi belli olmayan bitkilerdir.
\end{abstract}

Anahtar kelimeler: Yeni kayıt, fitocoğrafik bölge, endemik, flora.

\section{New Floristic Records for Some Squares from Turkey}

\begin{abstract}
In this study, 77 new plant taxa records for (A5, B7, B8, B9) are given for various squares. 13 of them are endemic (Bunium microcarpum (Boiss.) Freyn subsp. longiradiatum Hedge \& Lamond, Trigonosciadium intermedium Freyn \& Sint., Tanacetum eginense (Hausskn. ex Bornm.) Grierson, Heldreichia bupleurifolia Boiss. subsp. rotundifolia (Boiss.) Parolly, Nordt \& Mumm. var. rotundifolia, Hesperis pisidica Hub.-Mor., Anchusa limbata Boiss., Euonymus Iatifolius (L.) Mill. subsp. cauconis Coode \& Cullen, Astragalus nitens Boiss. \& Heldr., Salvia cilicica Boiss. \& Kotschy, S. euphratiaca Montbret \& Aucher ex Bentham var. leiocelycina (Rech.f.) Hedge, Stachys setifera C.A.Mey. subsp. lycia ( Gand.) Bhattacherje, Rhynchocorys odontophylla R. B. Burb \& I. Richardson, Acantholimon calvertii Boiss. var. glabrum Akaydın et Doğan). In 8 of the identified taxa, variations were detected, unlike their definition in flora. The distribution of 77 taxa according to phytogeographic region are as: Irano-Turanian 19, Mediterranean 10, European-Siberia 8, multi-region or un determinate 40.
\end{abstract}

Keywords: New record, phytogeographical region, endemic, flora.

\section{Giriş}

Türkiye bitki çeşitliliği; Anadolu'nun Asya ve Avrupa kıtaları arasında geçiş bölgesi olması, üç farklı fitocoğrafik bölgenin kesişim noktasında bulunması, farklı iklim tiplerinin etkisi altında oluşu ve engebeli topoğrafik yapısından kaynaklanmaktadır [1]. Türkiye'nin sahip olduğu bitki potansiyelinin tam olarak ortaya konması ancak detaylı çalışmalarla mümkündür. Türkiye'nin bilhassa doğu kesimleri floristik açıdan teferruatlı olarak çalışılamadığından çeşitli taksonların korolojik durumları tam olarak ortaya konulamamıştır. Diğer taraftan bazı taksonların değişen ekolojilerdeki varyasyon durumları yeterince incelenemeden tanımları hazırlanmış ve söz konusu taksonların varyasyon sınırları yeterince deskripsiyonlarına yansitılamamıştır [2].

\footnotetext{
*Sorumlu yazar: yyapar25@gmail.com

Geliş Tarihi: 14.02.2020, Kabul Tarihi: 18.05.2020
} 
$\mathrm{Bu}$ çalışmadaki veriler bir taraftan Türkiye'deki bitkilerin yayılış durumlarına katkıda bulunurken diğer taraftan revizyon çalışmaları için de önemli müracaat kaynağı olacaktır. Yapılan botanik gezileri esnasında toplan bitkilerin değerlendirilmesi sonucu A5, B7, B8 ve B9 karelerinde şimdiye kadar yayılışları bilinmeyen taksonların belirtilen lokalitelerde geliştikleri tespit edilmiştir. Ayrıca yayılışı belirlenen bitkilerin dahil oldukları fitocoğrafik bölge, ve bazı taksonlardaki varyasyon durumları ile ilgili değerlendirmelerde bulunulmuştur.

\section{Materyal ve Metot}

Çalışmamızın materyalini A5 (Çorum), B7 (Elazı̆̆), B8 (Bingöl, Erzurum) ve B9 (Bitlis) karelerinden topladığımız vasküler bitkiler oluşturmaktadır.

Toplanılan bitkiler; başta Türkiye florası [3-5] olmak üzere komşu ülke floraları [6-10] ve yapılan bazı revizyon çalışmaları [11] kullanılarak teşhis edilmiştir. Türkiye florası ve yayılışlarla ilgili çalışmalar [2, 12-51] taranarak yeni kayıt listesi oluşturuldu. Endemizim durumları, tehlike kategorileri, fitocoğrafik bölge elementleri, Türkçe isimler ve otör isimleri Türkiye bitkileri listesi (damarlı Bitkiler) [52] kitabı ve Türkiye Bitkileri Kırmızı kitabından [53] kontrol edilmiştir. Bitkiler herbaryum materyali haline getirilerek Bingöl Üniversitesi Fen-Edebiyat Fakültesi Biyoloji Bölümü Herbaryumu'nda saklanmaktadır.

Verilen yeni kayıt listesi APG III [54] sistemi dikkate alınarak alfabetik olarak verilmiştir. Lokalite bilgileri verilirken bulunduğu kare, il, ilçe, köy, mevkii, habitat, tarih, yükseklik, toplayıc1 numarası, endemizim durumu (END kısaltması ile), Tehlike kategorisi ve fitocoğrafik bölge elementi sırası izlenmiştir. Varyasyon belirlenen bazı taksonlara ait lokalite bilgilerinden sonra varyasyon gösteren yapılar ile ilgili açıklamalar yazılmıştır. Ayrıca bazı taksonlara ait resimler takson lokalite bilgilerinden sonra verilmiştir.

\section{Bulgular ve Tartışma}

\subsection{Floristik Liste (Yeni kayıt)}

\subsubsection{Apiaceae}

1. Anthriscus caucalis M.Bieb / deligımı

B7 Elazığ: Baskil ilçesi, Elazığ caddesi, bahçe kenarı,1200-1250 m, 30.04.2012, Behçet 7589

2. Bunium microcarpum (Boiss.) Freyn subsp. longiradiatum Hedge \& Lamond / yalık

B8 Bingöl: Merkez, mezarlık batısı, 2-3 km. taş ocağına giderken, step, 1100-1200 m, 25.05.2013, Behçet 8802, END., EN, Ir.-Tur. el.

3. B. pestalozzae Boiss. / esnek akser

B8 Bingöl: Bingöl, Sancak girişi köprü çevresi, çayır, 1564 m, 14.06.2014, Behçet 9944, E. Medit. el.

4. Malabaila secacul (Mill.) Boiss. subsp. secacul / davarotu

B8 Bingöl: Bingöl, Balıklıçay köyü girişi, yol kenarları, kayalık alan, 1613 m, 14.06.2014, Behçet 9882.

5. Oenanthe fistulosa L. / kazyak

B8 Bingöl: Elazı ̆ yolu üzeri, Mendo tesisleri güneyi, çayır, 1300-1400 m, 13.06.2015, Behçet 10710

6. P. tragium Vill. subsp. pseudotragium (DC.) V.A. Matthews. / yoz anason

B8 Bingöl: Genç ilçesi, Şam dağı, step, 1900-2000 m, 30.07.2015, Behçet 10860, Ir.-Tur. el.

7. Prangos peucedanifolia Fenzl / kaya çakşırı

B8 Bingöl: Bingöl, Balıklıçay köyü girişi, yol kenarları, kayalık alan, 1613 m, 14.06.2014, Behçet 9864. Ir.-Tur. el.

8. Torilis nodosa (L.) Gaertn. / boncuklu dercikotu

B7 Elazığ: Baskil ilçesi kuzeydoğusu Şefkat köyüne doğru demiryolu çevreler, Step, 900 m, 18.05.2013, Behçet 8681

9. Torilis tenella (Delile) Rchb.f. / zarif dercikotu

B8 Bingöl: Bingöl, Ilıcalar batısı, orman sahası orman açıları, 1100 m, 02.06.2013, Behçet 8864a

10. Trigonosciadium intermedium Freyn \& Sint. / öz şemsiyeotu

B8 Bingöl: Bingöl, Aşağıköy-Şaban köyleri arası, step, 1500-1600 m, 26.06.2014, Behçet 10010, END., EN, Ir.-Tur. el. 


\subsubsection{Asteraceae}

11. Anthemis haussknechtii Boiss. \& Reut. / çöl papatyası

B8 Bingöl: Bingöl, Balıklıçay köyüne 1-2 km kala yolun sağındaki vadi, dere kenarları, 1600-1620 m, 14.06.2014, Behçet 9840 .

12. A. pseudocotula Boiss. / acem papatyas1

B8 Bingöl: Bingöl Üniv. Kavşağı, yol kenarları, 1100-1150 m, 10.11.2015, Behçet 11049

13. Aster squamatus (Sprengel) Hieron / arsiz simpatı

B8 Bingöl: Bingöl Üniversitesi kampüsü, nemli alan, 1100-1150 m, 23.10.2017, Behçet 12509; B7

Elazığ: Keban ilçe merkezi, baraj çevresi, 700 m, 06.09.2018, Behçet 12894.

14. Conyza bonariensis (L.) Cronquist / çakalotu

B8 Bingöl: Bingöl Üniversitesi kampüsü, nemli alan, 1500-1200 m, 10.10.2014, Behçet 10371.

Yapraklar $18 \times 1.7 \mathrm{~cm}$ ye varlyor (Türkiye florasında $5-8 \times 0.5-1.5 \mathrm{~cm}$ ).

15. Erigeron nigromontanus Boiss. \& Buhse / tarla şifaotu

B8 Bingöl: Bingöl Aşağıköy aras1 12.km, 1500-1600 m, 31.07.2015, Behçet 10903, Ir.-Tur. el.

16. Inula germanica $\mathrm{L}$. / ekin andizotu

B8 Bingöl: Bingöl Üniv. Ziraat Fakültesi güneyi, yol kenarları, 1100-11500 m, 20.10.2015, Behçet 11027, Eu. Sib. el.

17. Lactuca saligna L. / deli marul

B8 Bingöl: Merkez, Sanayi sitesi çevresi, dere kenarları, 100-1050 m, 09.08.2014, Behçet 10271.

18. Matricaria chamomilla L. var. recutita (L.) Fiori / alman papatyas1

B7 Elazığ: Baskil ilçesi, Döküntü yerleri, 900 m, 18.05.2013, Behçet 8663b.

19. M. matricarioides (Less.) Porter ex Britton / kelkız çiçeği (Şekil 1)

B8 Erzurum merkez, eski terminal cami çevresi, nemli alan, 09.07.2019, 1900-1950 m, Behçet 8061;

B9 Bitlis: Tatvan ilçe merkezi, bahçe kenarları, 27.07.2017, 1670 m, Behçet 12409.

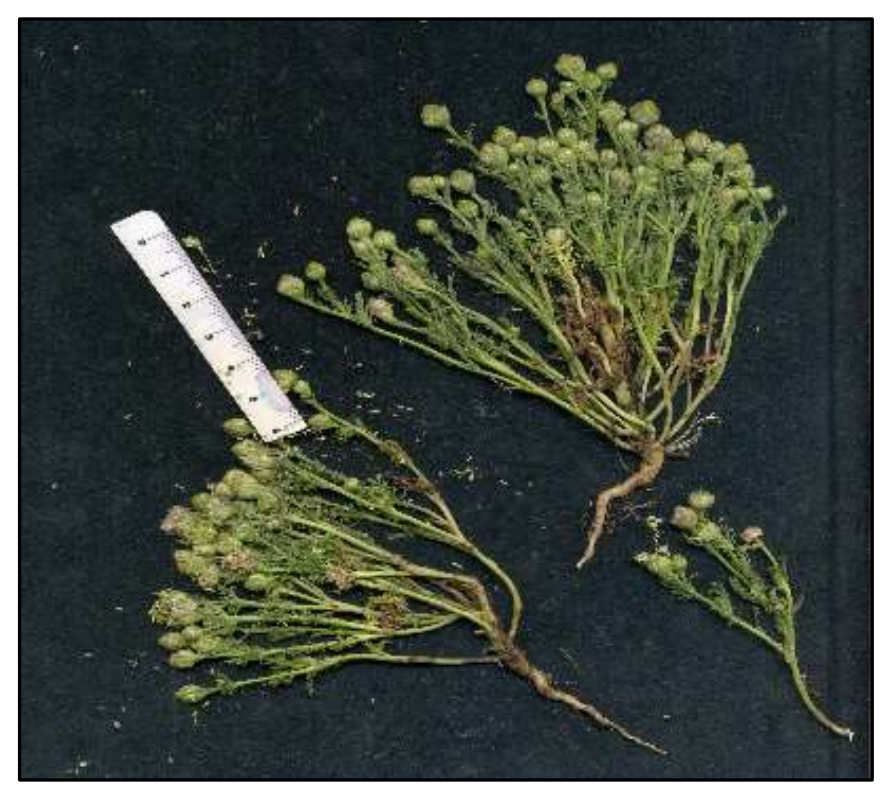

Şekil 1. Matricaria matricarioides

20. Picris hieracioides L. subsp. hieracioides / deli şiro

B8 Bingöl: Bingöl, Aşağıköy-Şaban köyleri arası, step, 1500-1600 m, 26.06.2014, Behçet 10011, Eu. Sib. el.

21. Tanacetum eginense (Hausskn. ex Bornm.) Grierson / has pireotu

B8 Bingöl: Bingöl, Şaban köyü güneyi, Metan dağı, step, 1900-2100 m, 06.06.2015, Behçet 10679, END., NT, Ir.-Tur. el.

22. Tragopogon porrifolius L. var. abbrevatus (Boiss.) Çoşkunçelebi \& M. Gültepe / çayır yemliği B8 Bingöl: Bingöl, Balıklıçay köyüne 1-2 km kala yolun sağındaki yamaçlar, kayalık, 1600-1660 m, 14.06.2014, Behçet 9763a 
23. Tripleurospermum parviflorum (Willd.) Pobed. / beybunik

B8 Bingöl: Bingöl, Bahçelievler mah. batıs1, step, 1200-1300 m, 10.05.2014, Behçet 9459

24. T. tenuifolium (Kit.) Fryn / saçaklı beybunik

B8 Bingöl: Bingöl, Sudüğünü köyü girişi, çayır, 1585 m, 14.06.2014, Behçet 9892a, Eu. Sib. el.

\subsubsection{Boraginaceae}

25. Anchusa limbata Boiss. / gövrek

B8 Bingöl: Bingöl Yelesen köyü kuzeyi, step, 1400-1600 m, 19.04.2015, Behçet 10435, END., CR, E. Medit. el.

\subsubsection{Brassicaceae}

26. Cardamine impatiens L. subsp. impatiens / sultan kadimotu (Şekil 2)

B8 Bingöl: Bingöl Aşağıköy, dere kenarları, 1500-1600 m, 06.06.2015, Behçet 10665, Eu. Sib. el.

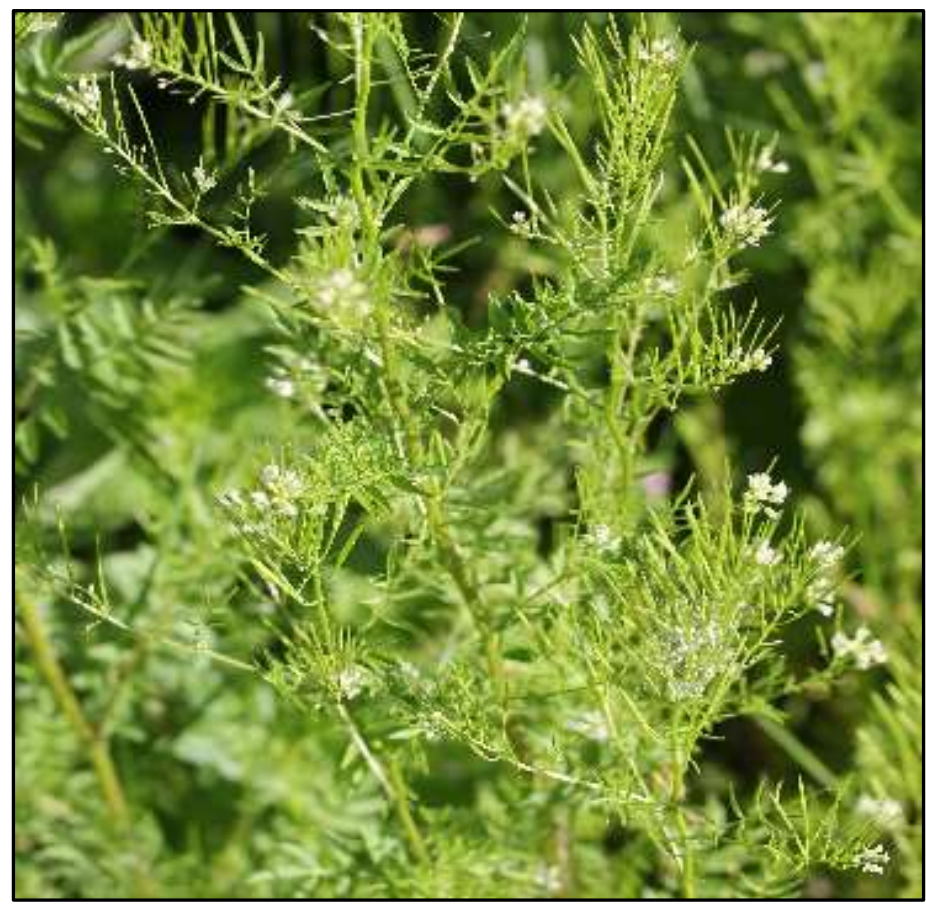

Şekil 2. Cardamine impatiens subsp. impatiens

27. Erysimum diffusum Ehreh. / Zarifeotu

B8 Bingöl: Bingöl Üniv. Kampüsü, lojman çevreleri, 1100-1150 m, 26.05.2014, Behçet 9530

28. Heldreichia bupleurifolia Boiss. subsp. rotundifolia (Boiss.) Parolly, Nordt \& Mumm. var. rotundifolia / oyalı topaç

B8 Bingöl: Bingöl, Yelesen köyü güneyi Kurt dağ1, Gerindal gölü çevresi, step, 2300-2450 m, 10.08.2014, Behçet 10333, END., LC, Ir.-Tur. el.

29. Hesperis pisidica Hub.-Mor. / dirmil y1ldızı

B8 Bingöl: Bingöl, Şaban köyü güneyi, yüksek dağ çayırları, 1700-1900 m, 06.06.2015, Behçet 10637, END., EN.

Örneğimizde sepal boyu 9-10 mm (Türkiye florasında 7-8 mm), Petal boyu $21 \mathrm{~mm}$ ye vartyor (Türkiye florasında $18 \mathrm{~mm}$ uzunluğunda deniyor)

30. Rorippa islandica (Oeder) Borbás subsp. islandica / acice

B8 Bingöl: Bingöl, Sanayi sitesi, dere kenarları, 1000-1050 m, 09.08.2014, Behçet 10278

31. Sisymbrium officinale (L.) Scop. / ergelen hardalı

B8 Bingöl: Merkez, Fen Lisesi çevresi, yol kenarlar1, 1100 m, 22.06.2014, Behçet 9990. 


\subsubsection{Caryophyllaceae}

32. Dianthus hymenolepis Boiss. / yamaç karanfili

B8 Bingöl: Bingöl, Kartal köyünü 1-2 km geçtikten sonra, yol kenarları, 1745 m, 14.06.2014, Behçet 9698, Ir.-Tur. el.

33. Sagina saginoides (L.) H.Karst. / yayla saginotu

B8 Bingöl: Bingöl, Yelesen köyü güneyi, Kurt dağı çevresi, dere kenarları, 1700-1900 m, 10.08.2014, Behçet 10357

34. Spergularia rubra (L.) J.Presl \& C.Presl / ramilotu (Şekil 3)

B8 Bingöl: Bingöl, Bingöl kuzeyi, sulama kanalı çevresi 1080-1150 m, 04.07.2015 Behçet 10816

Kapsül 3-3.5 mm uzunluğunda (Türkiye florasında 4-5 mm).

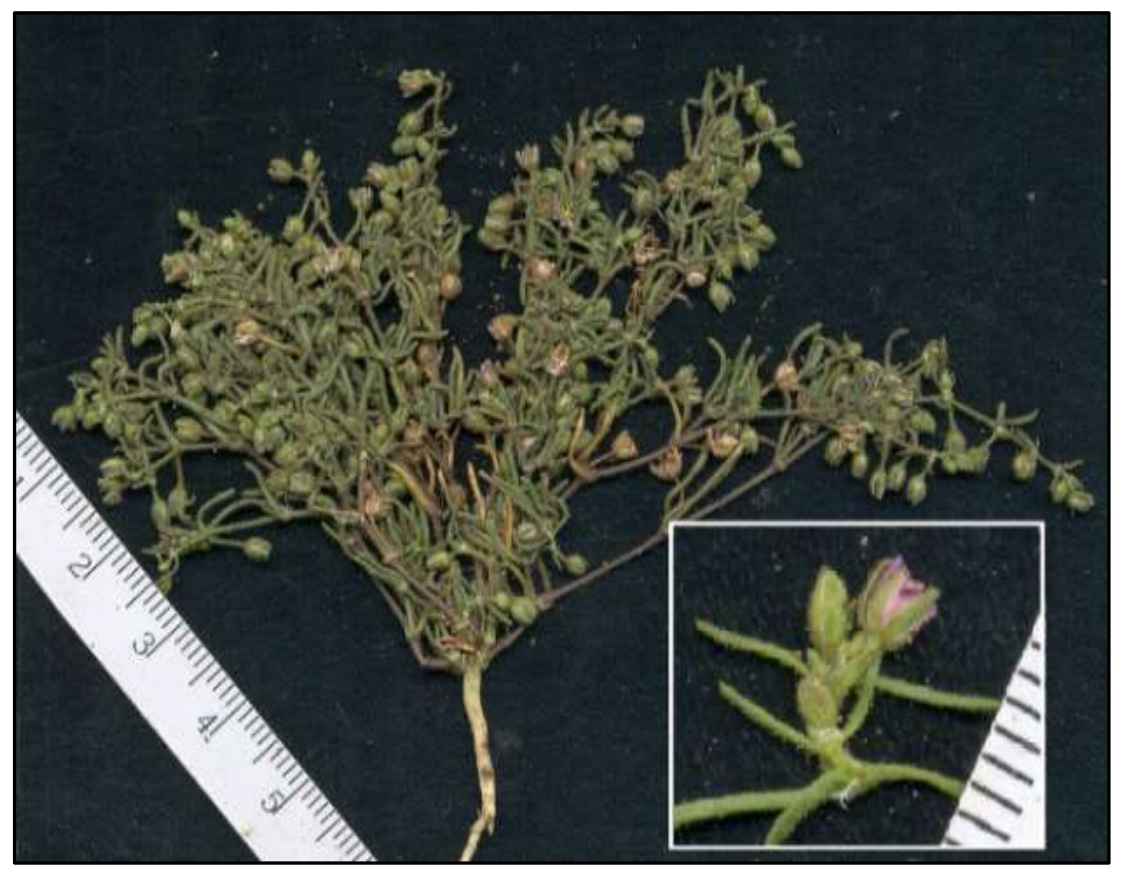

Şekil 3. Spergularia rubra

\subsubsection{Celastraceae}

35. Euonymus Iatifolius (L.) Mill. subsp. cauconis Coode \& Cullen / işyanotu

B8 Bingöl: Bingöl, Şaban köyü güneyi, yüksek dağ çayırları, 1700-1900 m, 06.06.2015, Behçet 10635, END., NT.

Yapraklar $16 \mathrm{~cm}$ 'ye varlyor (Türkiye florasinda $5-7 \mathrm{~cm}$ ).

\subsubsection{Crassulaceae}

36. Sedum confertiflorum Boiss. / küme koruğu

B8 Bingöl: Bingöl, Balıklıçay köyü girişi, kayalık, 1600m, 14.06.2014, Behçet 9884, E. Medit. el.

\subsubsection{Cyparaceae}

37. Carex disticha Huds. / atçimeni

B8 Bingöl: Bingöl, Sudüğünü köyü girişi, çayır, 1585 m, 14.06.2014, Behçet 9890a, Medit. el.

\subsubsection{Euphorbiaceae}

38. Euphorbia prostrata Aiton / yayık sütleğen

B8 Bingöl: Bingöl, Karşıyaka cami çevresi, 1100-1200 m, 22.10.2013, Behçet 10383 
39. E. serpens Kunth / ezil sütleğen

B8 Bingöl: Bingöl üniv. Rektörlük binası önü, yol kenarı, 1100-1150 m, 09.10.2015 Behçet 10990; Bingöl, Uydukent mah. Selahattin Eyyubi cami batısı, bahçe içi, 1100 m, 28.09.2011, Behçet 7460; B7 Elazığ: Baskil ilçesi Mezarlık çevreleri, 900 m, 23.10. 2012, Behçet 8366,

40. E. maculata L. / benli sütleğen

B8 Bingöl: Bingöl kuzeyi, sulama kanalı çevresi, 1080-1150 m, 04.07.2015 Behçet 10815; Bingöl, Uydukent mah. Selahattin Eyyübi cami batısı, bahçe içi, 1100 m, 28.09.2011, Behçet 7461; B7: Elazı̆̆ Baskil ilçesi Mezarlık çevreleri, 900 m, 23.10.2012, Behçet 8367

\subsubsection{Fabaceae}

41. Arachis hypogaea L. / yerfistı̆̆ 1

B8 Bingöl: Genç ilçesi yolu, Ziraat fakültesi uygulama arazisi, 1100-1150 m, 18.10.2015, Behçet 11022

42. Astragalus latifolius Lam. / geven yoncas1

B8 Bingöl: Bingöl, Çirişli-Şaban Köyleri arası yol kenarları, step alanlar, 1700-1900 m, 31.05.2014, Behçet 9619, Ir.-Tur. el.

43. A. nitens Boiss. \& Heldr. / parlak geven (Şekil 4)

B8 Bingöl: Bingöl, Şaban köyü güneyi, yüksek dağ çayırları, 1700-1900 m, 06.06.2015, Behçet 10623, END., LC, Ir.-Tur. el.

Stipül 3-5 mm uzunluğunda (Türkiye florasında $2 \mathrm{~mm}$ ), Brakte $4 \mathrm{~mm}$ (Türkiye florasında $2 \mathrm{~mm}$ ).

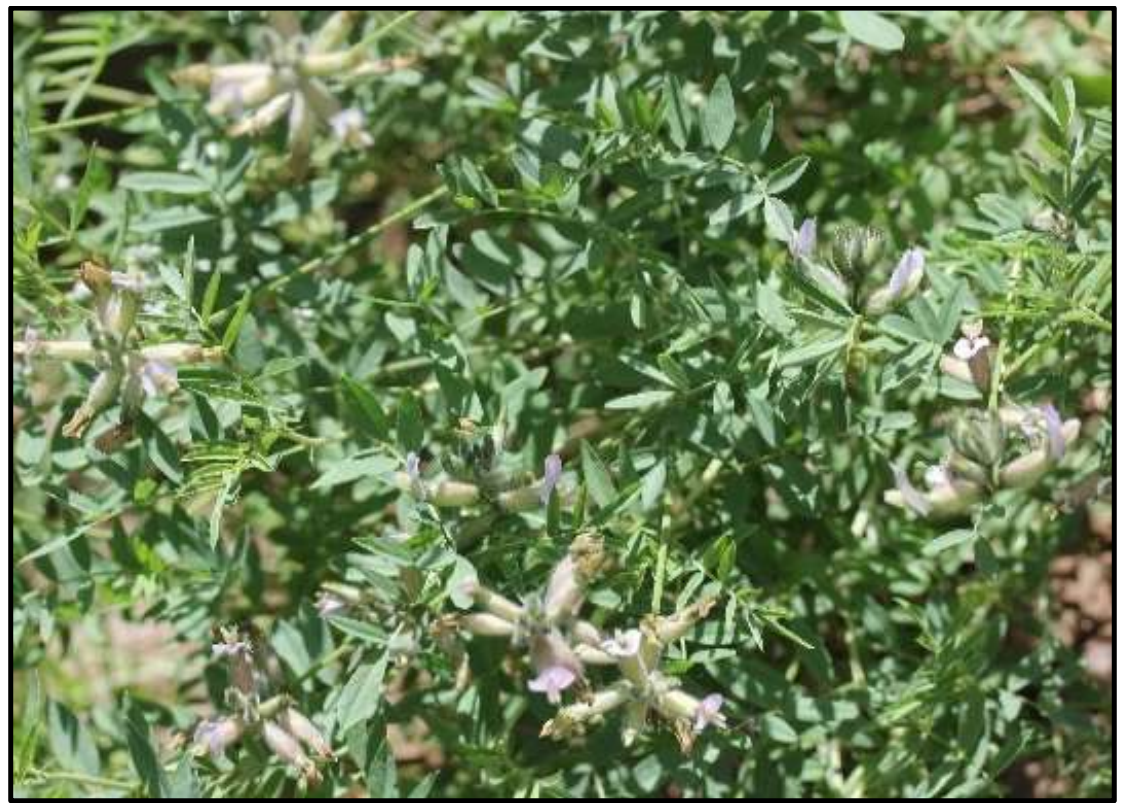

Şekil 4. Astragalus nitens

44. A. haarbachii Spruner / çarık geveni

B7 Elazığ: Baskil ilçesi eski demiryolu çevresi, Step, 900 m, 18.15.2013, Behçet 8692a

45. Onobrychis stenostachya Freyn subsp. sosnowskyi (Grossh.) Hedge / çoruh korungas1

B8 Bingöl: Bingöl, Aşağı köy-Ortaköy aras1 yol kenarları, step alanlar, 1400-1500 m, 31.05.2014, Behçet 9627, Ir.-Tur. el.

Bitki boyu $95 \mathrm{~cm}$ 'ye varlyor (Türkiye florasında $25-50 \mathrm{~cm}$ ). Yaprakçılar $27 x 5.5 \mathrm{~cm}$ boyutlarında (Türkiye florasında 7-20 x $1.5-4.5 \mathrm{~cm}$ )

46. Trifolium isthmocarpum Bort. / güdük yonca (Şekil 5)

B8 Bingöl: Bingöl Üniversitesi kampüsü, nemli alanlar, 1100-1150 m, 09.10.2015, Behçet 10981 


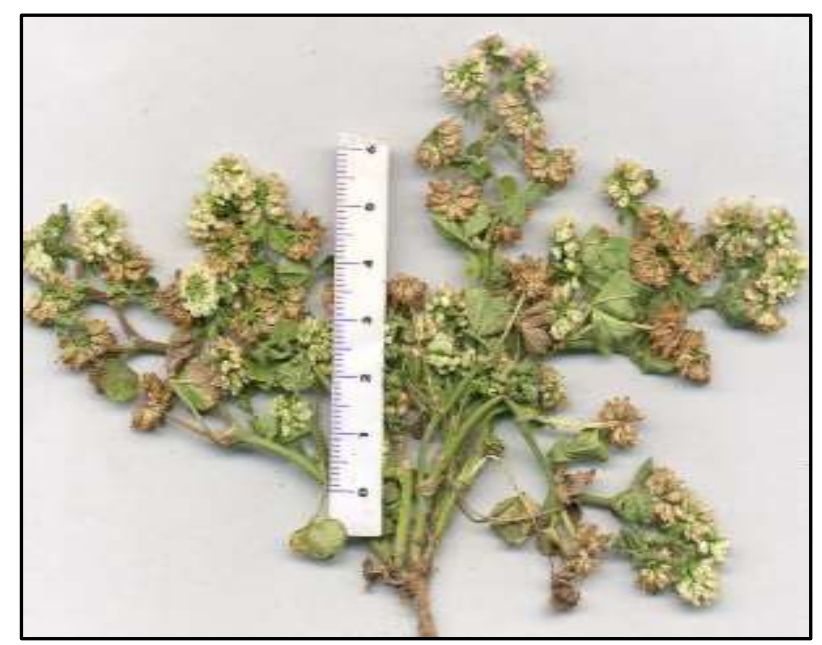

Şekil 5. Trifolium isthmocarpum

47. T. sylvaticum Gerard ex Lois. / orman üçgülü

Bingöl: Elazığ yolu üzeri, Mendo tesisleri güneyi, orman sahası, 1250-1400 m, 30.05.2015, Behçet 10601

48. Trigonella cylindracea Desv. / boru boyotu

B7 Elazığ: Baskil ilçesi, Şefkat köyü, Dere kenarları, 850 m, 27.10.2012, Behçet 8355, E. Medit. el.

\subsubsection{Gentianaceae}

49. Centaurium erythraea Rafn subsp. erythraea

B7 Elazığ: Baskil ilçesi, Yukarıkuluşağı köy 2 km kuzeyi, 1000-1100 m, 06.09.2015, Behçet 10965b, Eu. Sib. el.

\subsubsection{Lamiaceae}

50. Mentha pulegium L. / yarpuz (Şekil 6)

B8 Bingöl: Bingöl Üniv. Rektörlük binası önü, nemli alanlar, 1100-1150 m, 09.10.2015

Bitki nodlarda köklenmiş (Türkiye florasında bu durumdan bahsetmiyor) Rusya florasinda nodlarda köklendiği belirtiliyor.

Yaprak genellikle tüysüz (Avrupa florasında bilhassa yaprak alt yüzeyi tüysüz) Türkiye florasında tüy durumunun nemliliğe bă̆ll olarak çok değiştiğinden bahsediyor.

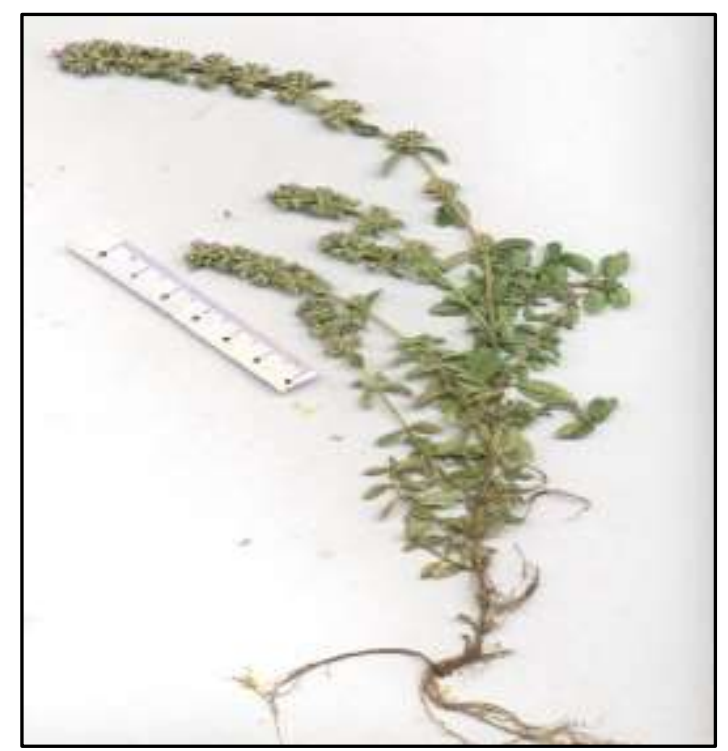

Şekil 6. Mentha pulegium 
51. Nepeta italica L. / eşekçay1

B8 Bingöl: Bingöl Üniversitesi kaplıcaları doğusu, yamaçlar, 1200-1300 m, 17.07.2014, Behçet 10220.

52. Salvia cilicica Boiss. \& Kotschy / narin galabor

B7 Elazığ: Baskil ilçesi, Yukarıkuluşağı köyü yukarı mah., step, 1000-1100 m, 28.06.2015, Behçet 10783, END., VU, Medit. el.

53. S. euphratiaca Montbret \& Aucher ex Bentham var. leiocelycina (Rech.f.) Hedge / fırat şalbas1 (Sekil 7)

B8 Bingöl: Bingöl, Yelesen köyü kuzeybatısı, kayalık yamaçlar, 1900-2200 m, 26.06.2014, Behçet 10071, END., NT, Ir.-Tur. el.

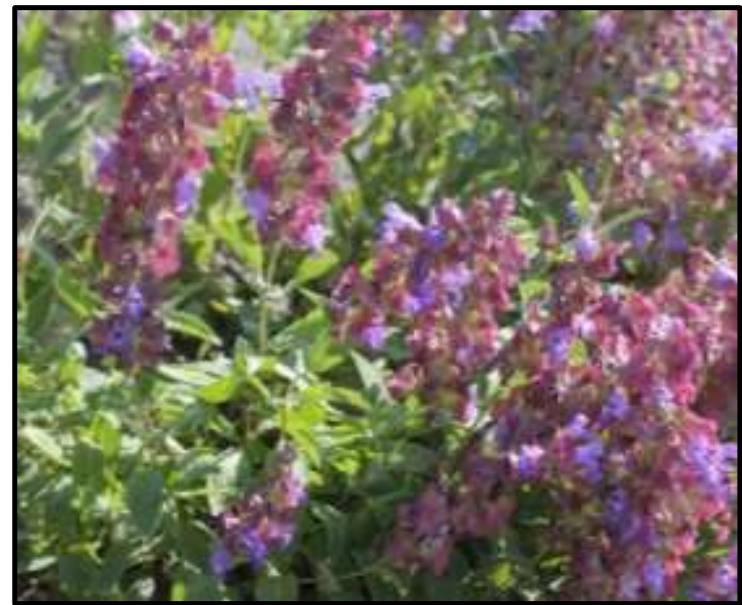

Şekil 7. Salvia euphratiaca var. leiocelycina

54. Scutellaria brevibracteata Stapf subsp. subvelutina (Rech.f.) Gauther \& Burdet / kadife kaside B8 Bingöl: Elazığ yolu üzeri, Mendo tesisleri güneyi, dere kenarları, 1250-1400 m, 30.05.2015, Behçet 10560, E. Medit. el.

55. Stachys longispicata Boiss. \& Kotschy

B7 Elazığ: Karlı köyü, Tarla, Tarla çevreleri, 800-900 m, 14.09.2012, Behçet 8340, Ir.-Tur. el.

56. S. setifera C.A.Mey. subsp. lycia ( Gand.) Bhattacherje / ince deliçay (Şekil 8)

B8 Bingöl: Bingöl, Çukurca köyü, meşe ormanı, nemli orman açıkları, 1630 m, 08.08.2015, Behçet 10914a, END., LC, Ir.-Tur. el.

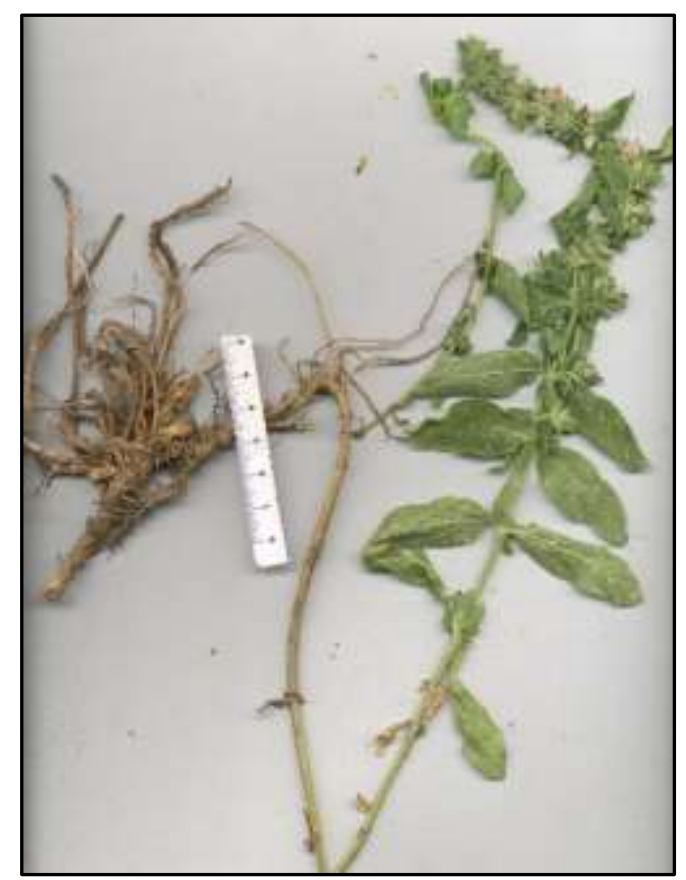

Şekil 8. Stachys setifera subsp. lycia 


\subsubsection{Liliaceae}

57. Gagea chlorantha (M.Bieb.) Schult. \& Schult.f. / güvercin ciğdemi

B8 Bingöl: Bingöl-Muş Karayolu Kervansaray dinlenme tesislerini geçtikten sonra, meşelik alan, 11001200 m, 11.03.2015, Behçet 10400 .

\subsubsection{Lythraceae}

58. Amannia auriculata Willd. / kulaklı amanotu

A5 Çorum: Osmancık ilçesi, Güvercinlik köyü, çeltik tarlaları, tarla kenarı, 435 m, 20.09.2016, Behçet \& Karasu 07

59. Lythrum virgatum L. / çamur aklarotu

B8 Bingöl: Bingöl kuzeybatıs1, sulama kanalı çevresi, 1080-1150 m, 04.07.2015, Behçet 10791, Eu. Sib. el.

\subsubsection{Onagraceae}

60. Epilobium lanceolatum Seb. \& Mauri / dilyakıs1

B8 Bingöl: Elazı̆̆ yolu üzeri, Mendo tesisleri güneyi, dere kenarları, 1250-1400 m, 30.05.2015, Behçet 10538

\subsubsection{Orobanchaceae}

61. Rhynchocorys odontophylla R. B. Burb \& I. Richardson / özge filburnu (Şekil 9)

B8 Bingöl: Bingöl, Şaban köyü güneyi, nemli çayır, 1700-1900 m, 06.06.2015 Behçet 10647, END., VU, Ir.-Tur. el.

Bitki boyu $150 \mathrm{~cm}$ 'ye varlyor (Türkiye florasinda $100 \mathrm{~cm}$ ), Yapraklar $10 \times 3.5 \mathrm{~cm}$ (Türkiye florasında $7 \times 1.5 \mathrm{~cm}$ ), Korolla alt dudağ $13 \mathrm{~mm}$ ' ye varlyor (Türkiye florasinda $8-10 \mathrm{~mm}$ ).

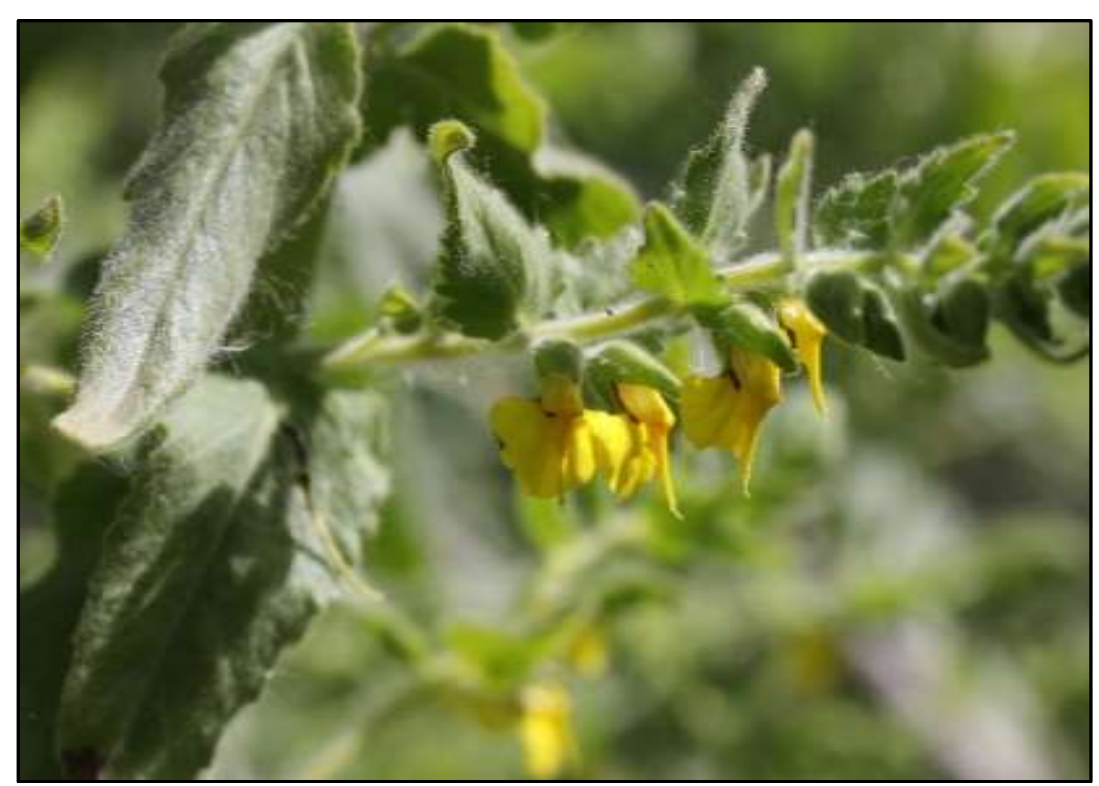

Şekil 9. Rhynchocorys odontophylla

\subsubsection{Plumbaginaceae}

62. Acantholimon calvertii Boiss. var. glabrum Akaydın et Doğan

B8 Bingöl: Bingöl Ortaçanak köyü kuzeyi, Kortev yaylası, step, 1850-1900 m, 08.08.2015, Behçet 10934. END., VU, Ir.-Tur. el. 


\subsubsection{Poaceae}

63. Bromus pumilio (Trin.) P.M. Sm. / bodur kılcan

B8 Bingöl: Bingöl, Yelesen köyü kuzeybatısı, kayalık yamaçlar, 1900-2200 m, 26.06.2014, Behçet 10085 ,

64. B. racemosum L. / salkım kilcan

B8 Bingöl: Bingöl, Sudüğünü köyü girişi, çayır, 1585 m, 14.06.2014, Behçet 9904b, Eu. Sib. el.

65. Festuca gigantea (L.) Vill. / koca yumak

B8 Bingöl: Bingöl, Yelesen köyü güneyi, Kurt dağı çevresi, dere kenarları, 1700-1900 m, 10.08.2014, Behçet 10358. Eu. Sib. el.

66. Hordeum distichon L. / ikili arpa

B8 Bingöl: Merkez, Çapakçur deresi, yürüyüş yolu, yamaçlar, 1050-1100 mi 04.06.2014, Behçet 9682

67. Hordeum murinum L. subsp. murinum / pisipisiotu

B8 Bingöl: Bingöl, Sudüğünü köyü girişi, çayır, 1585 m, 14.06.2014, Behçet 9896.

68. Loliolum subulatum (Banks \& Sol.) Eig / köseçim

B7 Elazığ: Baskil ilçesi eski demiryolu, çevresi, Step, 900 m, 18.05.2013, Behçet 8697a, Ir.-Tur. el.

\subsubsection{Polygonaceae}

69. Rumex obtusifolius L. subsp. subalpinus (Schur) Čelak / kökükızıl

B8 Bingöl: Bingöl, Karşıyaka cami çevresi, yerleşim alanları, 1100 m, 17.05.2014, Behçet 9974.

\subsubsection{Rosaceae}

70. Pyrus communis L. subsp. sativa (DC.) Hegi / armut

B8 Bingöl: Bingöl, Şaban köyü güneyi, yüksek dağ çayırları, 1700-1900 m, 06.06.2015, Behçet 10646.

71. Rosa elymaitica Boiss. \& Hausskn. ex Boiss. / gilan

B8 Bingöl: Merkez, Karşıyaka cami çevresi, yerleşim alanları, 1100 m, 17.06.2014, Baehçet 9987. Ir.Tur. el.

\subsubsection{Rubiaceae}

72. Galium debile Desf. / bataklık iplikçiği

B8 Bingöl: Elazığ yolu üzeri, Mendo tesisleri güneyi, çayır, 1300-1400 m, 13.06.2015, Behçet 10772, Medit. el

\subsubsection{Rutaceae}

73. Haplophyllum buxbaumii (Poir.) G.Don / sedef otu

B8 Bingöl: Bingöl Ormanardı köyü yol ayrımı, nemli kayalık yamaçlar, 1200 m, 10.10.2015, Behçet 11002b, Ir.-Tur. el.

\subsubsection{Scrophulariaceae}

74. Verbascum tripolitanum Boiss. / üç sığırkuyruğu

B8 Bingöl: Merkez, Fen lisesi çevresi, nemli alan, 1100 m, 07.06.2014, Behçet 9691, E. Medit. el.

\subsubsection{Solanaceae}

75. Physalis angulata L. / hışhış çiçeği

B8 Bingöl: Bingöl, Genç ilçesi yolu, Ziraat Fakültesi uygulama arazisi, 1100-1150 m, 18.10.2015, Behçet 1126

76. Solanum decipiens Opiz / eceavlusu

B8 Bingöl: Bingöl merkez kuzeyi, vadi içi, ekili alanlar, 1050- 1100 m, 31.10.2015, Behçet 11045 


\subsubsection{Tamaricaceae}

77. Tamarix parviflora DC. / deli 1lgin

B8 Bingöl: Bingöl, Bahçelievler mah. batısı, step, 1200-1300 m, 10.05.2014, Behçet 9464, Medit. el.

77 bitki taksonunun A5, B7, B8 ve B9 karelerindeki yayılışları ilk defa bu çalışmada verildi. 25 familyaya ait bu taksonların tamamı Angiospermae üyesidir. Bu bitkilerin 70'ü Dicotyledones ve 7'si Monocotyledones sınıflarına aittir. Verilen taksonlardan 13 (\%16.88)'ü (Bunium microcarpum (Boiss.) Freyn subsp. longiradiatum Hedge \& Lamond, Trigonosciadium intermedium Freyn \& Sint., Tanacetum eginense (Hausskn. ex Bornm.) Grierson, Heldreichia bupleurifolia Boiss. subsp. rotundifolia (Boiss.) Parolly, Nordt \& Mumm. var. rotundifolia, Hesperis pisidica Hub.-Mor., Anchusa limbata Boiss., Euonymus Iatifolius (L.) Mill. subsp. cauconis Coode \& Cullen, Astragalus nitens Boiss. \& Heldr., Salvia cilicica Boiss. \& Kotschy, S. euphratiaca Montbret \& Aucher ex Bentham var. leiocelycina (Rech.f.) Hedge, Stachys setifera C.A.Mey. subsp. lycia ( Gand.) Bhattacherje, Rhynchocorys odontophylla R. B. Burb \& I. Richardson, Acantholimon calvertii Boiss. var. glabrum Akaydın et Doğan) endemiktir. Endemik taksonların tehlike kategorileri şu şekildedir: 1'i CR (kritik), 3'ü EN (tehlikede), 3'ü VU (zarar görebilir), 3’ü NT (tehdide yakın), 3’ü LC (en az endişe verici).

Çalışmada belirlenen 77 taksonun fitocoğrafik bölgelere göre dağılımı şu şekildedir: İran turan 19 (\%24.67), Akdeniz 10 (\%12.99), Avrupa Sibirya 8 (\%10.39), çok bölgeli veya fitocoğrafik bölgesi bulunmayan 40 (\%51.95). Verilen yeni kayıt 8 takson bilinen tanımlarından farklı bazı varyasyonlar göstermektedir; bu durum yeni yayılış alanlarındaki farklı ekolojiye dayandırılabilir.

Çalışmada verilen taksonların bir kısmının Türkiye'nin doğusunda bugüne kadar yayılışı bilinmiyordu. Hatta Physalis angulata L.'nin 2000 yılına kadar Türkiye'nin herhangi yerinde yayılış1 olduğuna dair bilgi yoktu. Dünyada tabii olarak; Orta ve Güney Amerika ile Hindistan'da yayıllışı bilinen bu tür; ekolojik tölerans sınırları içinde olan Dünyanın değişik bölgelerine de zamanla yayılmıştır. Türkiye'de ise ilk defa Gönen ve arkadaşları tarafından [11] Çukurova bölgesinden (Adana, Osmaniye) yayılışı belirlenmiştir. Yakın zamanda da tarafımızdan Bingöl ve Diyarbakır'dan yayılışı belirlenmiştir. Bu durum; türün tölerans sınırlarının geniş olduğunu ve yayılış sınırlarını genişletmeye devam ettiğini göstermektedir.

Türkiye'de, nispeten 1lıman iklimli Batı Karadeniz-Marmara (A1 ve A5 karelerinden) bölgelerinde yayılışı bilinen Trifolium isthmocarpum Bort.'un denizden uzak ve karasal iklim etkisindeki Bingöl'den yayılışının belirlenmesi ilginç bir durumdur. Bu yayılışta Bingöl'e düşen yıllık yağışın $(950 \mathrm{~mm})$ etkisi yanında türün karasal iklim şartlarına töleranslı olduğunu da düşündürmektedir.

Türkiye'de şimdiye kadar sadece Akdeniz bölgesinde yayılışı bilinen Euphorbia prostrata Aiton ve $\boldsymbol{E}$. serpens Kunth'un Bingöl'deki yayılışında güneyden gelen sıcak iklim yapısının etkisi düşünülebilir.

Amannia auriculata Willd.'in Türkiye'deki yayılış1; şimdiye kadar sadece Türkiye'nin Gürcistan sınırına yakın Çoruh (Artvin) çevrelerinde biliniyordu. Yayılışı ile ilgili olarak; Türkiye Florası 4. cildinde verilen lokalite bilgilerinden sonra; "bu taksonun Türkiye'deki varlığının teyid edilmesi gerektiği" belirtilmektedir. Bu taksonun Çoruh çevresinden çok uzak, Osmancık (Çorum) ilçesi çeltik tarlalarında da yayılışı tarafımızdan kaydedildi. Dolayısı ile bu taksonun Türkiye'de yayılışının olduğu kesinleşmiş olmasının yanında; yayılış alanının da daha geniş olduğu bu kaydımız ile doğrulanmış oldu.

Aster squamatus (Sprengel) Hieron'un Türkiye Florasının 10. cildinde türün Kuzeydoğu Anadolu' da Çoruh, Hopa çevrelerinde yayılışı verilmiştir. Fakat daha sonra Türkiye'nin güneybatı ve batı kesimlerinde denizin yumuşatıcı etkisinin olduğu bazı habitatlarda da yayılışı belirlendiği gibi; karasal iklim özelliklerinin etkili olduğu Bingöl ve Keban(Elazı̆̆g)'nın sulak alan çevrelerinde de bu türün yayılışı tarafımızdan belirlendi olduğu belirlendi. Ayrıca bu güne kadar türün yayılışı deniz seviyelerinden 10 m'ye kadar olan yüksekliklerde biliniyordu hâlbuki Bingöl ve Keban'da 900-1150 m yüksekliklerde de bu bitkinin yayılışının olduğu ortaya kondu. Denizden çok uzak, yıllık ve günlük sıcaklık farklarının yüksek olduğu (karasal iklim özelliği) bu coğrafyada türün yayılışı ilginç bir durumdur.

Bugüne kadar Türkiye'de sadece Kars ve Artvin çevrelerinde yayılışı bilinen Matricaria matricarioides (Less.) Porter ex Britton'un [1] Erzurum ve Tatvan'da da yayılış1 belirlendi. Bu tespitimiz ile türün yayılış alanını genişlettiği anlaşılmaktadır. 


\section{Sonuç ve Öneriler}

Çalışma sonucunda yeni kare kaydı olarak verilen taksonların hem flora yazımında (taksonların yayılış sınırları ve tanımlarının doğru bir şekilde ortaya konması) hem de yapılan veya yapılacak olan revizyon ve flora çalışmalarında araştırmacılara kolaylık sağlaması ve daha fazla örnek inceleme imkanı sunması amacı ile herbaryum materyali haline getirilerek Bingöl Üniversitesi Fen-Edebiyat Fakültesi Biyoloji bölümü herbaryumunda saklanmaktadır.

\section{Yazarların Katkısı}

Yazarlar makaleye eşit oranda katkı sağlamıştır.

\section{Çıkar Çatışması Beyanı}

Yazarlar arasında herhangi bir çıkar çatışması bulunmamaktadır.

\section{Araştırma ve Yayın Etiği Beyanı}

Yapılan çalışmada araştırma ve yayın etiğine uyulmuştur

\section{Kaynaklar}

[1] Davis P.H. (ed.). 1965. Flora of Turkey and the East Aegean Islands. Vol. 1, Edinburgh Univ. Press, Edinburgh, U.K.

[2] Behçet L. 1999. New floristic records fort he grid squares B7 (Baskil-Elazı̆̆) and B9 (Van) from Turkey. Ot Sistematik Botanik Dergisi, 3 (1): 85-92.

[3] Davis P.H. (ed.). 1965-1985. Flora of Turkey and the East Aegean Islands. Vol. 1-9, Edinburgh Univ. Press, Edinburgh, U.K.

[4] Davis P.H., Mill. R.R., Tan K. (eds.) 1988. Flora of Turkey and the East Aegean Islands. Vol. 10, Edinburgh Univ. Press, Edinburgh, U.K.

[5] Güner A., Özhatay N., Ekim T., Başer K.H.C. 2000. Flora of Turkey and the East Aegean Islands. Vol.11, Edinburgh Univ. Press, Edinburgh, U.K.

[6] Towsend C.C., Guest E. 1966-1985. Flora of Iraq. Vol. 1-8, Baghdad: Ministry of Agriculture, Iraq.

[7] Rechinger K.H. 1965-1977. Flora Iranica. Vol. 1-9, Graz-Austria: Akademisch Drucku Verlangsanstalt.

[8] Zohary M. 1966-1986. Flora Palaestina. Vol. 1-4, Jerusalem Academic Press, Israel.

[9] Komarov V.L. (ed.) 1933-1964. Flora of the USSR. Vol. 1-30, Keter Press, Moskov.

[10] Tutin T.G., Heywood V.H., Burges N.A., Moore D.M., Valentine D.H., Walters S.M., Webb D.B. (eds.). 1964-1981. Flora Europaea. Vol. 1-5, Univ. Press, Cambridge.

[11] Podlech D., Zarre S. 2013. A taxonomic revision of the genus Astragalus L. (Leguminosae) in the old World. Vol. 1, Botany \& Zoology P.O. Box 1360, D-61453 Koenigstein, 391p, Germany.

[12] Gönen O., Yıldırım A., Uygur F.N. 2000. A new record for the flora of Turkey Physalis angulata L. (Solanaceae). Turkish Journal of Botany, 24 (5): 299-301.

[13] Behçet L. 1996. Türkiye'nin B7 (Baskil-Elazığ) ve B10 (Iğdır) karelerinden yeni floristik kayıtlar. Ot Sistematik Botanik Dergisi, 3 (1): 85-92.

[14] Behçet L., Yapar Y., Sinan A. 2014. Contribution to the flora of Turkey from B8 square (Bingöl, Elazığ/ Turkey). Biological Diversity and Conservation, 7 (3): 87-97.

[15] Çakılcıoğlu U., Civelek Ş. 2011. Flora of the region between copper mine and Tekevler village (Maden-Elazığ/Turkey). Biological Diversity and Conservation, 4 (1): 54-66.

[16] Donner J. 1990. Distribution maps to P.H. Davis. Flora of Turkey. Vol. 1-10, Linzer Biol. Beitr., 22 (2): 381-515.

[17] Kaya Y. 1995. Doğu Anadolu'dan B7 ve B8 kareleri( Erzincan-Tunceli) için yeni floristik kayitlar. Turkish Journal of Botany, 19: 153-158. 
[18] Kaya Y. 1997. New floristic records forthe grid squares A8 and B8. Turkish Journal of Agricultur and Forest, 23: 7-24.

[19] Kaya Y. 1997. Pasinler ovası ve çevresinin florasına katkılar. Ot Sistematik Botanik Dergisi, 4 (1): 9-26.

[20] Kılıç Ö., Bağcı E. 2011. Aşağıçakmak köyü ile Keban baraj gölü (Elazı̆̆) arasındaki sahanın floras1. Ot sistematik Botanik Dergisi, 18 (2): 79-130.

[21] Kılıç Ö. Yıldırımlı Ş. 2014. New nativ and cultivated species records fort he squares in the flora of Turkey. Ot Sistematik Botanik Dergisi, 21 (1): 47-68.

[22] Kılıç Ö. Yıldırımlı Ş. 2014. Dikme (kür) yaylası (Bingöl merkez) ve çevresinin fulorası. Ot Sistematik Botanik Dergisi, 21 (1): 69-126.

[23] Kılıç Ö. Yıldırımlı Ş., Kıranşan K. 2017. Yüzenadalar (Bingöl-Solhan) çevresinin fulorası. Ot Sistematik Botanik Dergisi, 24 (2): 131-172.

[24] Kurşat M., Civelek Ş., Türkoğlu İ. 2005. Elazığ, Baskil merkez ilçe-Altınkürek köyü (Keban) arasındaki yüksek sahanın florası. Fırat Üniversitesi Fen ve Mühendislik Bilimleri Dergisi, 17 (3): 541-557.

[25] Sinan A., Behçet L. 2014. The flora of Altıkardeş montain and its surroundings (Genç, Bingö1/ Turkey). Biological Diversity and Conservation, 7 (3): 97-116.

[26] Türkoğlu İ., Civelek Ş. 2005. Karga dağının (Elazığ) florası. Fırat Üniversitesi Fen ve Mühendislik Bilimleri Dergisi, 17 (2): 370-399.

[27] Türkoğlu İ., Civelek Ş. 2006. Gözeli ve Kavak ovalarının (Elazığ) florası. Fırat Üniversitesi Fen ve Mühendislik Bilimleri Dergisi, 18 (1): 11-23

[28] Türkoğlu İ., Civelek Ş. 2008. Kuşakçı dağının (Elazığ) florası. Ot sistematik Botanik Dergisi, 15 (1): 71-96.

[29] Türkoğlu İ., Civelek Ş., Kurşat M. 2009. Kamışlık dağı (Elazığ) florası. Fırat Üniversitesi Fen Bilimleri Dergisi, 21 (2): 123-138.

[30] Yildırımlı Ş. 1997. The chorology of the Turkish species of Acanthaceae, Aceraceae, Aizoaceae, Amaranthaceae and Anacardiaceae families. Ot Sistematik Botanik Dergisi, 4 (1): 125-130.

[31] Yildırımlı Ş. 1997. The corology of the Turkish species of Apiaceae family. Ot Sistematik Botanik Dergisi, 4 (2): 105-128.

[32] Yıldırımlı Ş. 1999. The chorology of the Turkish species of Asteraceae family. Ot Sistematik Botanik Dergisi, 6 (2): 75-123.

[33] Y1ldırıml Ş. 2000. The chorology of the Turkish species of Boraginaceae family. Ot Sistematik Botanik Dergisi, 7 (2): 257-272.

[34] Y1ldırıml Ş. 2001. The chorology of the Turkish species of Brassicaceae, Buddlejaceae and Buxaceae families. Ot Sistematik Botanik Dergisi, 8 (1): 141-171.

[35] Yildırıml Ş. 2001b. The chorology of the Turkish species of Cactaceae, Callitrichaceae, Calycanthaceae and Campanulaceae families. Ot Sistematik Botanik Dergisi, 8 (2): 157-171.

[36] Yildırımlı Ş. 2002. The chorology of the Turkish species of Caryophyllacaea, Casuarianeceae, Celestraceae, Cerathophyllacaeae and Cercidiphyllaceae families. Ot Sistematik Botanik Dergisi, 9 (2): 175-199.

[37] Yildirımlı Ş. 2003. The chorology of the Turkish species of Chenopodiaceae, Cistaceae, Convolvulaceae, Cornacea and Corylaceae families. Ot Sistematik Botanik Dergisi, 10 (1): 203215.

[38] Yildırımlı Ş. 2003. The chorology of the Turkish species of Crassulaceae, Cucurbitaceae, Cuscutaceae and Cynocrambaceae families. Ot Sistematik Botanik Dergisi, 10 (2): 249-263.

[39] Yildırıml Ş. 2005. The chorology of the Turkish species of Fabaceae family. Ot Sistematik Botanik Dergisi, 12 (1): 117-170.

[40] Yildırıml Ş. 2006. The chorology of the Turkish species of Gentianaceae, Geraniaceae. Gesneriaceae, Globulariaceae and Grossulariaceae. Ot Sistematik Botanik Dergisi, 13 (1): 183194.

[41] Yildırımlı Ş. 2008. The chorology of the Turkish species of Lamiaceae family. Ot Sistematik Botanik Dergisi, 14 (1): 151-198.

[42] Yıldırımlı Ş. 2008. The chorology of the Turkish species of Lauraceae, Lentibulariaceae, Linaceae, Lobeliaceae, Lorantaceae and Lythraceae families. Ot Sistematik Botanik Dergisi, 14 (2): 180-197. 
[43] Y1ldirıml Ş. 2008. The chorology of the Turkish species of Oleaceae, Onagraceae, Orobanchaceae and Oxalidaceae families. Ot Sistematik Botanik Dergisi, 15 (2): 151-166.

[44] Yıldırımlı Ş. 2009. The chorology of the Turkish species of Pulumbaginaceae, Polemoniaceae, Poligalaceae, Polygonaceae, Portulacaceae, Primulaceae, Proteaceae and Punicaceae families. Ot Sistematik Botanik Dergisi, 16 (2): 189-211.

[45] Y1ldırıml Ş. 2011. The chorology of the Turkish species of Rosaceae family. Ot Sistematik Botanik Dergisi, 18 (1): 191-235.

[46] Yıldırımlı Ş. 2011. The chorology of the Turkish species of Rubiaceae and Rutaceae families. Ot Sistematik Botanik Dergisi, 18 (2): 173-204.

[47] Yıldırıml Ş. 2012. The chorology of the Turkish species of Scrophulariaceae family. Ot Sistematik Botanik Dergisi, 19 (1): 151-211.

[48] Yıldırımlı Ş. 2012. The chorology of the Turkish species of Simaroubeaceae, Solanaceae, Staphyleaceae, Sterculiaceae and Styracaceae families. Ot Sistematik Botanik Dergisi, 19 (2): 151-165.

[49] Y1ldırımlı Ş. 2013. The chorology of the Turkish species of Tamaricaceae, Theaceae, Thymelaeaeceae, Tiliaceae, Trapaceae, Tropaeolaceae, Ulmaceae and Urticaceae families. Ot Sistematik Botanik Dergisi, 20 (1): 157-170.

[50] Yıldırımlı Ş. 2015. The chorology of the Turkish tepaloid Monocotyledones species of Liliaceae Juss. s.l. family. Ot Sistematik Botanik Dergisi, 22 (1-2): 67-119.

[51] Yıldırımlı Ş. 2017. The chorology of the Turkish non tepaloid Monocotyledones species of Cyperaceae Juss., Juncaceae Juss. and Poaceae Barnhart (Gramineae) families. Ot Sistematik Botanik Dergisi, 24 (2): 173-235.

[52] Güner A., Aslan S., Ekim T., Vural M., Babaç MT. 2012. Türkiye bitkileri listesi (Damarlı bitkiler). Nezahat Gökyiğit Botanik Bahçesi ve Flora Araştırmaları Derneği Yayını, İstanbul.

[53] Ekim T., Koyuncu M., Vural M., Duman H., Aytaç Z., Adıgüzel N. 2000. Türkiye bitkileri kırmızı kitabı. Türkiye Tabiatını Koruma Derneği ve Yüzüncü Y1l Üniv., Ankara.

[54] Haston E., Richardson J.E., Stevens P.F., Chase M.W., Harris D.J. 2009. The linear angiosperm phylogeny group (LAPG) III: A linear sequence of the families in APG III. Botanical Journal of Linnean Society, 161: 128-131. 